\title{
Le «Messie mystique» et la Bourse d'Amsterdam, le 3 mai 1666
}

\author{
Carsten L. WILKE* \\ S. L. Steinheim-Institut für deutsch-jüdische Geschichte, Duisburg
}

Comment un obscur kabbaliste de Smyrne, se proclamant Messie en 1665, a$\mathrm{t}$-il pu répandre dans la diaspora juive une euphorie de fin du monde? Comment les «croyants» de Sabbataï Tsevi ont-ils pu trouver un sens et une logique dans un Messie qui ne put jouer son rôle de roi rédempteur que pendant trois semaines, un Messie fugitif et incarcéré (février 1666), un Messie apostat (septembre 1666), puis un Messie mourant paisiblement sous le nom de Mehmet Pacha, maître d'hôtel en Bosnie (1676)?

Voilà quelques-unes des énigmes qu'un jeune professeur de la jeune Université Hébraïque de Jérusalem chercha à résoudre quand, en 1927, il partit en mission pour chercher dans les fonds manuscrits européens des renseignements sur les origines de ce mouvement messianique et les tournures paradoxales et antinomiques qu'il allait prendre. Gershom Scholem, l'envoyé de Jérusalem, conçut son hypothèse quand il étudia, à Oxford, le Maguen Avraham, l'un des premiers textes théologiques du sabbataïsme, et quand il attribua cet écrit à un marrane portugais, Abraham Michaël Cardoso. En effet, le mouvement sabbataïste apparaît à une époque où des milliers de juifs clandestins du Portugal étaient revenus publiquement à la foi de leurs ancêtres suite à de longues générations de clandestinité, voire d'apostasie pure et simple.

Les sectateurs du mouvement messianique, auraient-ils vu dans l'apostasie de leur Messie l'apothéose d'un drame tout semblable à celui qu'ils avaient eux-mêmes vécu? Passant par Paris, Scholem s'empressa à exposer ses observations à son ami Walter Benjamin: pendant une soirée au Café du Dôme, il ne fut question que de Cardoso et des marranes ${ }^{1}$. On peut supposer qu'à cette occasion, Scholem commença à interpréter sa découverte à la lumière du sens «ré-

\footnotetext{
*wilke@phil-fak.uni-duesseldorf.de

${ }^{1}$ Gershom Scholem, Walter Benjamin - die Geschichte einer Freundschaft (Frankfurt 1975), p. 171-172 (p. 159 de la traduction française, Walter Benjamin, histoire d'une amitié, Paris 1982).
} 
volutionnaire» que Benjamin avait donné au messianisme juif et qu'il conçut le portrait-type du croyant sabbataïste: un marrane pour qui la rédemption devait passer par l'aliénation la plus complète. Scholem consacra immédiatement à Cardoso un article dans la revue de Martin Buber ${ }^{2}$, et il élabora dix ans plus tard son hypothèse principale: c'est dans l'expérience particulière des judéo-convertis, nous dit-il, dans la «psychologie marrane» donc, que se trouve enfouie «la clef concernant la plupart des questions du sabbataïsme et les soubassements les plus profonds de la nouvelle doctrine» ${ }^{3}$.

La thèse, aussi séduisante soit-elle, repose sur des bases documentaires plutôt faibles. Certes, Cardoso était descendant de nouveaux-chrétiens, et il allait rapprocher lui-même, en 1668, le destin de Sabbataï de celui des crypto-juifs portugais ${ }^{4}$. Mais sa théologie de l'apostasie du Messie lui vint de seconde main; le principal artisan en fut l'ashkénaze Nathan de Gaza. Déjà Haïm Wirszubski avait souligné l'importance de ce kabbaliste ${ }^{5}$, et Scholem lui-même, au terme d'une étude rigoureuse des sources, a retracé les étapes à partir desquelles Nathan conçut son enseignement hétérodoxe. Le même regard critique conduisit Scholem à reconnaître, dans son magnum opus sur Sabbataï Tsevi ${ }^{6}$, que le désenchantement dans les communautés judéo-portugaises à la suite de l'apostasie du prétendant fut immédiat et quasi global. Il n'y a en effet aucune commune mesure entre les groupuscules sabbataïstes clandestins à Amsterdam et à Livourne et les mouvements de masse qu'on observa parmi les communautés plus traditionnelles de l'Empire Ottoman ou de Pologne. Même l'attribution du Maguen Avraham à Cardoso s'était avérée fausse ${ }^{7}$. Ces observations ont amené

${ }^{2}$ Gershom Scholem, «Über die Theologie des Sabbatianismus im Lichte Abraham Cardosos», Der Jude 10 (1928), 123-139; réimprimé dans Gershom Scholem, Judaica I (Frankfurt 1963), pp. 119-146.

${ }^{3}$ «La rédemption par le péché. Pour une compréhension du sabbataïsme» (en hébreu), Keneset 2 (1937), p. 259, 369; traduction française -établie sur la traduction anglaise- dans Gershom Scholem, Le Messianisme juif (Paris 1974), p. 158-159. Scholem exprime la même idée dans Les grands courants de la mystique juive (Paris 1951), p. 327-328.

${ }^{4}$ Lettre d'avril 1668, texte hébreu chez Abraham H. Freimann (éd.), Sammelband kleiner Schriften über Sabbatai Zebi und dessen Anhänger (Berlin 1912), p. 88; allégué par ScHOLEM, «La rédemption par le péché», p. 359.

${ }^{5}$ «La doctrine sabbataïste sur l'apostasie du Messie» (en hébreu), Zion 3 [NS] (1937/38), 215-227.

${ }^{6}$ Shabbetai Zevi and the Shabbetaian Movement during His Lifetime (en hébreu) (Tel-Aviv 1957), réédité en 1974 et en 1987. L'auteur a retravaillé ce livre pour la traduction anglaise de 1971, qui a été traduite en français par A. Nouss et M.-J. Jolivet, Sabbatai Tsevi. Le Messie mystique, 1626-1676 (Paris 1983).

${ }^{7}$ Scholem avait avancé cette attribution dans «La rédemption par le péché», p. 361, et dans sa publication du texte dans Kobez Al Jad 12 (1938), 121-155. Wirszubski a voulu donner la paternité 
Scholem à désavouer sa thèse «marraniste». L'impact des «nouveaux juifs» sur le mouvement sabbataïste fut, décida-t-il, plutôt négligeable ${ }^{8}$. Tout au plus futce «un canal important» ${ }^{9}$ par lequel certaines idées gnostiques chrétiennes pouvaient intégrer la mythologie des sabbataïstes. Mais la thèse fascinante élaborée lors d'une conversation parisienne en 1927 survécut au désavœu de son auteur; elle a fait une réapparition éclatante dans la recherche récente. Dans des travaux de seconde main, elle a acquis une quasi-unanimité ${ }^{10}$.

Je ne voudrais pas ici retracer les motivations qui ont amené Scholem à accentuer pour un temps la soif de rédemption des groupes les plus aliénés du judaïsme. Ce n'est pas non plus mon propos de m'interroger sur la question de savoir pourquoi sa première explication semble de nouveau s'imposer aux historiens de nos jours. Je voudrais limiter mon étude au déroulement du mouvement parmi les juifs portugais à Amsterdam pendant sa première phase, quand le problème de l'apostasie ne se posait pas encore. Une source nouvellement découverte permettra de cerner de plus près l'attitude des juifs portugais à l'égard $\mathrm{du}$ «Messie mystique» en désignant nommément pour la première fois les chefs

du Maguen Avraham à Nathan lui-même. Dans son livre de 1957, Scholem revint sur ses propos et il attribue le traité à un troisième sabbataïste, R. Abraham Perets, dont le nom figure dans un des manuscrits (Shabbetai Zevi [hébreu], p. 701). Scholem s'est corrigé encore une fois depuis pour rendre le texte à Cardoso (Sabbataï Tsevi, français, p. 791). Plus tard, Yehuda Liebes a démontré que celui-ci ne saurait en aucun cas en être l'auteur (Kirjath Sepher 55 [1980], 603-616).

${ }^{8}$ Shabbetai Zevi (hébreu), p. 682; français, p. 774.

${ }^{9}$ Shabbetai Zevi (hébreu), p. 681. L'adjectif «important» disparaît de la version retravaillée (voir la trad. française, p. 773).

${ }^{10}$ La thèse a été acceptée notamment par I. S. RÉvaH, «Les marranes», RÉJ 118 (1959/60), p. 73, par Haim H. Ben-SASSON, Histoire du peuple juif (en hébreu) (Tel- Aviv 1969, 5éd. 1976), t. II, p. 303, et par Yosef H. Yerushalmi, De la Cour d'Espagne au ghetto italien: Isaac Cardoso et le marranisme au XVII siècle, traduit par A. Nouss (Paris 1987), p. 281-282. António J. Saraiva va jusqu'à parler d'une seule et même «flambée messianique qui eut son principal foyer à Amsterdam et qui culmina à Smyrne», voir son «António Vieira, Menasseh ben Israel et le Cinquième Empire», Studia Rosenthaliana 6 (1972), p. 56. Les éditeurs des Cahiers Spinoza 3 (hiver 1979-1980), p. 143-145, ont reproduit les citations de Scholem parmi les textes-clef pour la compréhension des juifs portugais en général et de Spinoza en particulier. Dans les travaux plus récents, l'accueil est encore plus chaleureux; voir, par exemple, Stephen SнArot, Messianism, Mysticism and Magic (Chapel Hill 1983), p. 101-102; Richard H. Popkin, Isaac La Peyrère (1596-1676). His Life, Work and Influence (Leiden 1987), p. 94-95; Gabriel AlBIAC, La sinagoga vacía. Un estudio de las fuentes marranas del espinosismo (Madrid 1987), p. 44; Edgar Morin, préface à l'ouvrage Les Juifs d'Espagne, histoire d'une diaspora, éd. H. Méchoulan (Paris 1992), p. iv. Seul Julio Caro Baroja a jugé que le «marranisme» multiforme invoqué par Scholem est un passe-partout conceptuel, capable d'expliquer tout sans rien expliquer; Julio CARO BAROJA, Los judios en la España moderna y contemporánea (Madrid, 2éd. 1978), t. I, p. 527. 
de file de l'opposition anti-sabbataïste. Ce document nous fera découvrir l'empreinte que l'histoire exceptionnelle des juifs portugais a effectivement laissée dans la réaction de certains, mais ce fut d'une façon exactement contraire aux suppositions de Scholem et de ses successeurs.

La chronologie du mouvement messianique d'Amsterdam ${ }^{11}$ nous montre à quel point celui-ci fut une réaction aux directives venues d'Orient. En novembre 1665 , les premiers messages, encore mal confirmés, apprirent aux juifs d'Amsterdam la proclamation messianique de Sabbataï Tsevi et ses miracles. Les rabbins de la communauté, Isaac Aboab, Moïse Raphaël d'Aguilar et Isaac Naar, furent immédiatement gagnés. Leur collègue Jacob Sasportas raconte qu'ils organisèrent une vaste propagande, amplifiée par les soins de l'opulent marchand Abraham Pereira. Celui-ci venait alors de livrer aux presses un ouvrage de dévotion et de pénitence, littérature bienvenue pour la préparation aux grands évènements. Mais, chose curieuse, les trois rabbins signèrent leurs lettres d'approbation le 3 et le 7 janvier 1666 sans se permettre la moindre allusion à la rédemption qu'ils croyaient imminente ${ }^{12}$.

Pour s'affirmer au grand jour, le mouvement messianique attendit le moment où les autorités rabbiniques d'Orient, celles de Jérusalem et du Caire notamment, lui donnèrent leur caution. Le 5 février, les missives du prétendant sur les changements liturgiques provoquèrent l'euphorie générale. Ce jour même, l'entrepreneur colonial João d'Ilhão demanda à partir en Terre Sainte avec cinquante familles ${ }^{13}$. Le samedi 6 février, des fonds pour l'embarquement prévu furent réunis par trois autres membres importants de la communauté: Moïse Curiel alias Jerónimo Nunes da Costa, Isaac Baruch et un certain Moreno ${ }^{14}$. De nouvelles épîtres du Messie sur le renouvellement de la prophétie furent reçues le 10 mars; et le lendemain, la synagogue portugaise adapta sa liturgie aux évé-

${ }^{11}$ Sauf mention contraire, tous les renseignements qui suivent sont tirés de la synthèse de Scholem, Shabbetai Zevi (hébreu), p. 284-288, 429-451, 640-643, 768-770, français, p. 351-356, 503-528, 731-734, 868-869. Uri Kaufmann et Michael Studemund-HaLÉvy ont publié une ample documentation sur l'impact du mouvement dans la communauté-sœur à Hambourg, «Dokumente zur Affaire Shabtai Zvi in Hamburg», dans Die Sefarden in Hamburg, éd. M. Studemund-Halévy (Hamburg 1994), t. I, p. 225-265.

${ }^{12}$ Ce livre a été réédité par Henry Méchoulan, Hispanidad y judaísmo en tiempos de Espinoza. Edición de La Certeza del Camino de Abraham Pereyra (Salamanca 1987); voir p. 96-105.

${ }^{13}$ R.G. Fuks-Mansfeld, De Sefardim im Amsterdam tot 1795: aspecten van een joodse minderheid in een Hollandse stad (Hilversum 1989), p. 113-114.

${ }^{14}$ Scholem ne réussit pas à identifier ces personnages. Selon le fichier manuscrit d'Abraham de Mordechai Vaz Dias (Aliassen, aux Archives municipales d'Amsterdam), Isaac Baruch fut le nom juif de Baltasar Alvares Nogueira; le deuxième personnage était probablement le docteur Jacob Moreno. 
nements survenus. Le 15 mars, Abraham Pereira et Isaac Naar partirent pour la Turquie à la rencontre du sauveur.

Des rumeurs sur l'internement de Sabbataï commencèrent à circuler dès le mois de mai. Mais étant donné la dépendance complète des juifs d'Amsterdam vis-à-vis des autorités rabbiniques d'Orient, qui gardaient alors un silence embarrassé, l'atmosphère d'attente à Amsterdam perdura encore tout l'été. Le 10 août, jour de deuil en mémoire de la destruction du temple de Jérusalem, fut transformé en une fête de rejouissance, Moïse ben Gidéon Abudiente publiant au Danemark son tract Fin de los días à cette occasion. Le 24 septembre encore, deux académies talmudiques d'Amsterdam envoyèrent des lettres de soumission à Sabbataï Tsevi.

Le 15 de ce mois, celui-ci avait déjà «pris le turban». Mais une fois de plus, la nouvelle, connue à Amsterdam dès les premiers jours de novembre, ne fut acceptée qu'un mois plus tard, quand les autorités méditerranéennes l'eurent finalement reconnue. Dès ce moment, le revirement fut complet et immédiat; à partir du 12 décembre, les écrits de propagande sabbataïste furent interdits par les mêmes personnes qui les avaient diffusés peu de semaines auparavant. Les chefs des «croyants» de jadis s'appliquaient maintenant de toute leur force à la répression du mouvement ${ }^{15}$.

Dans son étude, Scholem veut donner raison au rabbin Sasportas, qui tient à souligner l'adhésion quasi unanime des juifs d'Amsterdam au mouvement sabbataïste ${ }^{16}$. Mais il semble que ce chroniqueur engagé, qui tient à dramatiser sa lutte solitaire contre le fol enthousiasme, n'a pas pu s'empêcher d'exagérer sur ce point. L'historien Da Silva Rosa trouva des indications montrant que les autorités juives d'Amsterdam avaient à affronter tout un cercle de récalcitrants obstinés ${ }^{17}$. Pour protester, ces «incroyants» avaient cessé d'acquitter leurs impôts communautaires; ils ne fréquentaient même plus la synagogue. Leur poids économique fut apparemment suffisant pour que cette mesure inquiétât les di-

\footnotetext{
${ }^{15}$ Ce bouleversement d'attitude provoqué par la nouvelle d'apostasie est souligné par le chroniqueur David Franco Mendes, «Memorias do estabelecimento e progresso dos judeos portuguezes e espanhoes nesta famosa cidade de Amsterdam: a Portuguese Chronicle of the History of the Sephardim in Amsterdam up to 1772», éd. B. N. Teensma, L. Fuks et al., Studia Rosenthaliana 9, 2 (1975), 1-233, voir p. 67. L'apostasie de Sabbataï Tsevi était considérée comme la preuve majeure de l'inauthenticité de ses prétentions messianiques; voir Henry Méchoulan, Etre juif à Amsterdam au temps de Spinoza (Paris 1991), p. 132-133.

${ }^{16}$ Shabbetai Zevi (hébreu), p. 430, français, p. 505. Cette unanimité est soulignée également par Yosef Kaplan, From Christianity to Judaism: The Story of Isaac Orobio de Castro (en hébreu) (Jerusalem 1983), p. 183.

17 Jacob S. da Silva Rosa, «De indruk van Sabbatai Tsebi, den valsen Messias, te Amsterdam (1666)», De Vrijdagavond, numéro zéro (11 janvier 1924), p. 5-6; ID., Geschiedenis der Portugese Joden te Amsterdam. 1593-1925 (Amsterdam 1925), p. 89.
} 
rigeants; ceux-ci obtinrent de la municipalité la permission de ramener les «incroyants» à l'obéissance en les mettant au herem (ban) de la communauté.

Une source découverte par l'historien israélien Yosef Kaplan nous montre que ce herem n'a pas manqué d'être proclamé. Mais ce fut dans des circonstances assez particulières, car cette mise au ban visait des anonymes.

Le 28 Nissan 5426 [i. e. lundi, le 3 mai 1666] fut proclamé, solennellement et du pupitre synagogal, le herem contre celui ou ceux ayant rédigé - ou contribué à rédiger- un certain papier imprimé à propos des incroyants qui fut ven$\mathrm{du}$ aujourd'hui à la Bourse [certo papel q' se estampou, e vendeo neste dia na bolsa; $s^{r e}$ os incredulos] et qui fit outrage contre l'honneur du nom de Dieu, béni soit-il, et contre l'espérance en la venue de notre Messie. [...] Quiconque aura la moindre connaissance ou un soupçon bien fondé à propos de ceux ayant commis ce délit devra se manifester dans les vingt-quatre heures, à défaut de quoi il sera exclu de la même façon que ses complices ${ }^{18}$.

La date de ce décret est révélatrice. Il intervint au moment même où l'on reçut à Amsterdam les premières nouvelles sur l'arrestation du Sabbataï Tsevi, dont le navire avait été arraisonné sur la mer de Marmara ${ }^{19}$. Le Oprechte Haerlemse Saterdagse Courant du samedi, $1^{\text {er }}$ mai 1666 , semble avoir été la première gazette néerlandaise à rapporter l'arrestation ${ }^{20}$. Un ou plusieurs «incroyants» durent imprimer aussitôt un tract ou un pasquin anonyme contre leurs adversaires, qui circulait le lundi 3 mai parmi les gens de la Bourse. Le document découvert par Kaplan nous permet à peine de spéculer sur l'ampleur de cette opposition, sur ses acteurs, sur ses buts et sur ses manifestations. Mais la méticulosité des services de renseignement de l'Inquisition espagnole nous permettra de retrouver la piste du «papier» perdu.

Pendant trente ans, les sbires du Saint-Office avaient rodé à la porte de Fernão Montezinos, grand banquier portugais à la Cour de Madrid. Plusieurs

\footnotetext{
${ }^{18}$ Publié dans Yosef KaPLan, «The Attitude of the Leadership of the Portuguese Community in Amsterdam to the Sabbatian Movement, 1665-1671» (en hébreu), Zion 39 (1974), 198-216, p.

${ }^{19}$ Les «croyants» n'ont évidemment rien fait pour divulguer cet incident, intervenu le 6 février 1666. Ce n'est que le 5 avril qu' un proche du prétendant communique la nouvelle dans une lettre de Constantinople. Le contenu de celle-ci nous est connu par le témoignage de Thomas Chappell de Londres, qui avait reçu la nouvelle le 12 mai d'Amsterdam par le millénariste huguenot Pierre Serrarius.

${ }^{20}$ Jetteke VAN WIJK, «The Rise and Fall of Shabbetai Zevi as Reflected in Contemporary Press Reports», Studia Rosenthaliana 33 (1999), p. 24.
} 204. 
fois incarcéré et torturé, le magnat s'était finalement enfui à Anvers où il mourut en $1659^{21}$. Il avait réussi à transmettre ses biens à ses trois fils Bartolomeu, Manuel et Henrique Montezinos ${ }^{22}$, venus de Séville pour diriger la filiale madrilène. Si tout le monde savait qu'ils travaillaient avec des juifs de l'étranger, il fut impossible de déceler la moindre faille dans leur pratique catholique, quoique tout Madrid semble les avoir espionnés. L'Inquisition réunit un dossier des accusations disponibles ${ }^{23}$, mais la prudence des trois victimes désignées ne lui facilitait pas la tâche. Le 18 mai 1665, les inquisiteurs étaient réduits à établir procès-verbal de l'observation d'une adolescente excitée qui affirma avoir observé Bartolomeu en train de se gratter le nez pendant la messe. «J'en fus si scandalisée», dit la jeune Madrilène, «que je l'aurais abattu sur le champ, si seulement j'avais eu un poignard sur moi» ${ }^{24}$.

Le soir du $1^{\text {er }}$ juin 1666, se présente au Saint-Office un déclarant plus sérieux, Adrián González Brizeño, vingt et un ans, comptable de profession, «Castillan, homme d'honneur et chrétien de vieille souche». C'est le secrétaire de l'entreprise Montezinos Frères. Ce matin-là, ses patrons étant partis pour un voyage d'affaires, il avait ouvert lui-même le courier venu des Pays-Bas. Il était tombé alors sur une enveloppe sans nom d'envoyeur. Cette lettre mystérieuse ne contenait qu'un petit papier imprimé dont la teneur lui sembla suspecte.

L'indiscrétion du secrétaire ne fut pas non plus d'un grand secours pour les inquisiteurs dans les recherches qu'ils menaient contre le chef d'entre-

${ }^{21}$ Cette personnalité est l'objet d'une biographie de Bernardo LóPEZ Belinchón, Honra, Libertad y Hacienda: Hombres de negocios y judíos sefardies (Madrid 2001).

${ }^{22}$ Cédule royale du 5 mai 1655, citée par Daniel M. Swetschinski, «The Spanish Consul and the Jews of Amsterdam», dans Texts and Responses. Studies Presented to N. N. Glatzer on the Occasion of his Seventieth Birthday by his Students (Leiden 1975), p. 160.

${ }^{23}$ Madrid, Archivo Histórico Nacional, section Inquisición (dorénavant: AHN, Inq.), libro 1131, fols. 77-105. Ce dossier fut ouvert le 9 avril 1663. Il a été utilisé par Markus SchreIBer, Marranen in Madrid 1600-1670 (Stuttgart 1994), p. 356-357, 395; et par nous dans Jüdischchristliches Doppelleben im Barock. Zur Biographie des Kaufmanns und Dichters Antonio Enríquez Gómez (Frankfurt 1994), p. 406-407. Plus récemment, l'écho du sabbataïsme en Espagne a retenu l'attention de LóPez Belinchón, Honra, Libertad y Hacienda, p. 411; Id., «Familia, negocios y sefardismo», dans Familia, religión y negocio. El sefardismo en las relaciones entre el mundo ibérico y los Países Bajos en la Edad Moderna, éd. J. Contreras et al. (Madrid 2003), p. 343-363 (voir p. 360, 363); et ID., «Los criptojudíos españoles y Sabatai Zevi», dans Política y cultura en la época moderna (Cambios dinásticos, milenarismos, mesianismos y utopías), éd. A. Alvar, J. Contreras et J. I. Ruiz (Alcalá de Henares 2004), p. 697-704, voir surtout p. 703-704 sur notre document.

${ }^{24}$ AHN, Inq., lib. 1131, fols. 97-98, du 9 janvier 1663. 
prise. L'étrange feuille imprimée fut réunie au dossier ${ }^{25}$. En la lisant, on se rend compte que c'est justement le pamphlet «sur les incroyants» qui avait été distribué à la Bourse d'Amsterdam, le lundi 3 mai au matin. En voici le texte.

LISTA de los Señores Incredulos, y enjermados de la muy noble y Lucida Sinagoga Portuguesa de Amsterdam.

Iuan Nuñez Enriquez.

Elias Señor Coronel.

Jacob Netto.

Henrico Mendez da Silva.

...... de Sousa.

Abraham Pimentel.

Joseph Franco.
Samuel Pinto.

...... Carvalho.

Antonio Lopez Suasso.

Moyses de Silva.

Sebastin Cotinho.

Andreas Nuñes.

Iacques Nuñez Belmonte.

Y otros muchos que tanto por su autoridad, y otros por su bajeça no van nombrados.

Estos Señores por no haver creydo con todo animo que el $S^{r}$ Sabetha Seby, fuese el verdadero, y tantos atrasados años deseado Messia, y solamente fueron en opinion que segun la ley Judaica lo pudiera ser, y en ninguna manera lo quisieron admitir con tanta seguridad como algunos que dicen ser tan verdadero como la Deidad del sumo, y eterno Jehova, los Señores muy sabios, y ecelentes Rabies de sudicha lucida Sinagoga, queriendo hacer justicia de tan enorme incredulidad, resoluieron poner los nombres sudichos en un papel, y quemarlos como enjermados del Mamar, y malditos de la suma autoridad de dicho poderoso Rey Seby, y no recivirlos mas en su dicha Sinagoga antes que huviessen passado el Malcut penitencia mayor que la del habito de St. Benito, agora pero llegando la funebre nueva del mal suceso de dicho malaventurado Sabetha Seby, lo pusieronle en oluido hasta la venida de otro mas siguro, y finxido Messia que serà el nono deste nombre.

${ }^{25}$ AHN, Inq., lib. 1131, fols. 101-105 avec le titre: Autos sobre vn papel impreso que bino en correo de flandes con sobre escrito para D. Barme Montesinos. Le petit imprimé est devenu le fol. $103 \mathrm{r}$ du recueil. 
Sabetha Seby se embarcò ${ }^{26}$ en servicio del Gran Turco, $Y$ va escriviendo ${ }^{27}$ en el agua con una pluma de pino.

Expliquons d'abord les trois termes hébreux dont les auteurs de cette pièce ont ponctué leur satire, termes techniques qui sont typiques de l'administration des communautés judéo-portugaises. Le mot mamar est évidemment une coquille pour ma'amad, désignant le conseil de sept administrateurs annuellement élu qui gouvernait les affaires intérieures de la communauté. Parmi les mesures disciplinaires à sa disposition fut le ban ou herem; ainsi le mot enjermado, terme hébreu encadré de deux morphèmes espagnols, désigne une personne exclue temporairement de la communauté ${ }^{28}$. Dans des cas particulièrement graves, un enjermado ne pouvait faire lever le ban qu'en subissant la peine du fouet public ou malqût, prévue par le Talmud pour de nombreuses classes de délits ${ }^{29}$. Dans la plupart des cas, le ban ne fut donc pas une mesure d'exclusion mais de discipline, visant réintégrer un dissident tout en le mettant au pas.

Dans son vocabulaire et dans sa teneur, notre petite affiche est la parodie d'un décret de herem proclamé à la synagogue. Nous pouvons déterminer son modèle littéraire avec précision, car il n'y eut, pendant le deuxième tiers du XVII ${ }^{\mathrm{e}}$ siècle, que deux excommunications d' «incroyants» ${ }^{30}$ : la première fut promulguée le 27 juillet 1656 contre le jeune marchand Bento de Spinoza et la seconde, le 4 février 1658, contre le docteur Juan de Prado. Les deux hommes avaient pareillement nié l'authenticité de la Révélation scripturaire, mais il faut noter que le ban contre Prado n'était pas assorti des malédictions solennelles que nous trouvons dans celui de Spinoza, car les dirigeants espéraient encore renvoyer le docteur vers une communauté juive d'outremer ${ }^{31}$. Seul Spinoza, qui refusait

${ }^{26}$ Corrigé à la main. Dans l'imprimé: «Imbarco».

${ }^{27}$ Corrigé à la main. Dans l'imprimé: «escriendo».

${ }^{28}$ Ces mots sont étrangers à l'espagnol commun du XVII ${ }^{\mathrm{e}}$ siècle, à telle enseigne que López Belinchón a cru devoir corriger le texte en enfermar (rendre malade), dans LóPEz BELINCHón, «Los criptojudíos españoles», p. 703.

${ }^{29}$ Le Talmud contient tout un traité (Makkot, «coups») traitant des modalités de l'application de cette peine, appuyé sur Deutéronome 25,1-3. Yosef Kaplan a démontré à partir des archives que la peine du fouet fut toujours en usage à Amsterdam au XVII ${ }^{\mathrm{e}}$ siècle, en Yosef Kaplan, «The Social Function of the Herem in the Portuguese Jewish Community of Amsterdam in the Seventeenth Century», Dutch Jewish History, ed. J. Michman (Jerusalem 1984), p. 142.

${ }^{30}$ KaPLAN, «The Social Function of the Herem», p. 137.

${ }^{31}$ Israel S. Révah, Spinoza et le Dr Juan de Prado (Paris - La Haye 1959), p. 58-60, traduction française p. 28-30. 
catégoriquement de se rétracter, fut exclu et frappé de malédiction ${ }^{32}$. En faisant allusion à une formulation employée une décennie auparavant ${ }^{33}$, les auteurs du pamphlet semblent comparer leur cas à celui des «athées» qui avaient alors fait scandale. Le sens de ce procédé littéraire est évident: les rabbins sont accusés d'avoir perdu tout sens de la mesure en voulant assimiler l'incroyance en un certain «Monsieur Sabetha Sevy» à l'athéisme pur et dur.

Mais l'attitude que cette petite satire attribue aux rabbins caricaturés est pour le moins contradictoire. D'une part, on leur fait fulminer contre les «incroyants» les malédictions réservées à l'athée impénitent; d'autre part, ils incitent les inculpés à subir la pénitence. L'union de l'exclusion et du châtiment corporel manque de logique, mais les auteurs du pamphlet réussissent ainsi à utiliser deux sujets particulièrement pénibles pour leurs adversaires. En effet, dans une société où l'honneur, la bonne réputation, était considéré comme étant le suprême bien, la peine humiliante du fouet scandalisait beaucoup de spectateurs ${ }^{34}$. Cela notamment à la suite de la fin tragique d'Uriel da Costa, qui, s'étant soumis au malqût pour faire lever le ban qui pesait contre lui, souffrit tant de cet opprobre qu'il se suicida en 1640. On trouva auprès de son corps un manuscrit où il

${ }^{32}$ Cette malédiction exceptionnelle distingue le ban contre Spinoza du «herem ordinaire»; Jonathan I. IsRaEL, «Philosophy, Commerce and the Synagogue: Spinoza's expulsion from the Amsterdam Portuguese Community in 1656», dans Dutch Jewry: Its History and Secular Culture, éd. J. I. Israel et R. Salverda (Leiden 2002), p. 125-139 ; voir p. 137.

${ }^{33}$ Les mots «como enjermados de Mamar, y malditos de la suma autoridad de dicho poderoso Rey Seby» singent la malédiction prononcée contre Spinoza: «nos enhermamos, apartamos e maldisoamos e praguejamos a Baruch de Espinoza, com consentim[en]to del D[io] B[endito] e consentim[en]to de todo este K[ahal] K[ados]», cité par RÉvaH, Spinoza, p. 58. L'anathème employé provient de la compilation hébraïque $K o l$ Bô (vers 1300); il fut traduit en espagnol par Saül Lévi Mortera, rabbin d'Amsterdam, en 1618. Le néologisme enjermar n'y figure pas encore: «nos escomulgamos y esconjuramos, apartamos, destruimos, maldezimos y maldezimos aquella tal persona con la intencion del Sancto Bendito y con la intencion deste kaal kados») (voir Herman P. SAlomon, «La vraie excommunication de Spinoza», dans Forum Litterarum: Miscelânea de estudos literários, linguísticos e históricos oferecida a J. J. van den Besselaar, éd. H. Bots et M. Kerkhoff [Amsterdam - Maarssen 1984], p. 185, 197).

${ }^{34} \mathrm{C}$ 'est probablement une des raisons pour laquelle Sixtus Amama, recteur de l'université de Franeker, traduit le traité talmudique Makkot comme appendice à sa traduction du traité Sanhedrin, texte fondamental de l'eschatologie rabbinique. Voir Duo tituli thalmudici, Sanhedrin et Maccoth: Quorum ille agit de Synedriis, judiciis, suppliciis capitalibus Ebraeorum; Hic de poena falsi testimonii, exsilio \& asylis, flagellatione: cum Excerptis ex utriusque Gemara, versa, \& annotationibus, depromtis maximam partem ex Ebraeorum commentariis, illustrata à Ioanne Coch Bremensi (Amsterdam 1629). Sur le véritable auteur de cette traduction voir Peter van RoodEN, Theology, Biblical Scholarship and Rabbinical Studies in the Seventeenth Century (Leiden 1989), p. 81-82. 
dénonce le traitement inhumain dont il fut la victime ${ }^{35}$. Johannes Müller, pasteur à Hambourg, s'était saisi de ce texte en 1644 pour exiger que la «discipline ecclésiastique» soit retirée aux représentants des communautés juives ${ }^{36}$. Bref, les auteurs du pamphlet n'ont pas hésité à se saisir d'un topos de l'antijudaïsme protestant afin de renforcer leur accusation principale: en intervenant dans l'affaire sabbatéenne, les rabbins et les dirigeants communautaires ont dépassé les limites de leur autorité.

Nous remarquons que la peine du fouet est directement assimilée au port du Sambenito, le «sac béni» par antiphrase, habit infâmant qui devait être porté par les condamnés des Inquisitions espagnole et portugaise ${ }^{37}$. C'est précisément le docteur Prado, vivant alors à Anvers, qui aimait soutenir que les mesures disciplinaires de la communauté juive étaient illégitimes et qu'elles suscitaient une cruauté comparable à celle des Inquisitions ibériques ${ }^{38}$. Si les opposants au ma 'amad se comparent aux hérétiques Spinoza et Prado, ce n'est pas seulement pour mettre en relief l'absurdité d'une telle comparaison. Pour expliquer cette étrange ambiguïté à l'égard du pouvoir religieux, il nous faut jeter un coup d'oeil sur les personnes qui ont tenu à s'afficher comme «incroyants» dans la liste soi-disant anonyme du 3 mai 1666.

Une première observation s'impose tout de suite: les quatorze personnes ayant inscrit leurs noms sur cette parodie «anonyme» appartinrent au noyau le plus riche parmi les juifs d'Amsterdam ${ }^{39}$. António Lopes Suasso (c. 1607-1685) fut même le plus fortuné de tous, à en juger par les impôts qu'il payait ${ }^{40}$. Lui,

\footnotetext{
${ }^{35}$ «Judicet nunc qui haec audierit, quale esset spectaculum, videre hominem senem, sortis non abjectae, naturaliter verecundum super omnem modum, in concione publicâ coram omnibus tam viris quam mulieribus \& pueris nudatum, \& flagro caesum ex mandato judicum [...] Unum inter multa miror, \& verè mirandum est, quomodo possunt Pharizaei inter Christianos agentes uti tantâ libertate, ut etiam judicia exerceant [...] Certè hoc ignominiosum est, \& quod tolerari non debuit in civitate liberâ, quae profitetur homines in libertate \& pace tueri, \& tamen non tuetur a Pharizaeis injuriis», dans Die Schriften des Uriel da Costa, éd. C. Gebhardt (Amsterdam etc. 1922), p. 114, 123.

${ }^{36}$ Johannes MülLER, Judaismus oder Jüdenthum, das ist: Ausführlicher Bericht von Jüdischen Volcks Unglauben, Blindheit und Verstockung (Hamburg 1644), passage reproduit par Yoshuah BARJITZCHAK, Uriël da Costa (Den Haag 1962), p. 125-126.

${ }^{37}$ Sur les origines de ce mot, voir Américo CASTRO, «Sambenito», RFE 15 (1928), 179-181.

${ }^{38}$ Voir les sources publiées par Israel S. RÉvAH, «Aux origines de la rupture spinozienne: nouveaux documents sur l'incroyance dans la communauté judéo-portugaise d'Amsterdam à l'époque de l'excommunication de Spinoza», RÉJ 123 (1964), p. 371, 391, 406.

${ }^{39}$ Ce fait est souligné par LóPez Belinchón, «Los criptojudíos españoles», p. 703.

${ }^{40}$ Herbert I. Bloom, The Economic Activities of the Jews of Amsterdam in the 17th and 18th Centuries (Williamsport, Penn. 1937), p. 204. La biographie de cet entrepreneur a été écrite par
} 
João Nunes Henriques, Henrique Mendes da Silva, Moïse da Silva et Andrês Nunes figurent dans les actes de la Banque d'Amsterdam parmi les douze ou quinze entrepreneurs les plus considérables de leur communauté ${ }^{41}$. Lors de la guerre franco-néerlandaise, Lopes Suasso et un Jacob Coutinho -peut-être identique ou apparenté au Sebastião Coutinho mentionné dans la liste- furent parmi les plus importants munitionnaires de leur pays ${ }^{42}$.

Il semble que tous les autres signataires furent également des négociants. On est, certes, tenté d'identifier «Sousa» avec l'anti-sabbataïste le plus notoire d'Amsterdam, le docteur Abraham Gomes da Sousa, qui avait été jadis, aux Pays Bas espagnols, le médecin personnel du Cardinal-Infant Ferdinand ${ }^{43}$. Mais le pamphlet semble non pas se rapporter à cet individu isolé, mais à une famille, très probablement aux frères Simão \& Luis Rodrigues Sousa (à la synagogue, ce furent les frères Abravanel Sousa). Cette «noble» famille, dont De Barrios atteste l'orgueil de lignage ${ }^{44}$, est la quatrième entreprise juive dans le régistre de la Compagnie des Indes Occidentales en 1674, où António Lopes Suasso occupe la première place ${ }^{45}$.

Jacob ZwarTs, «De Haagsche familië Lopes Suasso», dans J. Zwarts, Hoofdstukken uit de geschiedenis der joden in Nederland (Amsterdam 1929), p. 212-227, et par Daniel M. SwETschinsKi et Loeki Schönduve, De familie Lopes Suasso, financiers van Willem III (Zwolle 1988); voir aussi Peter Busss, «Sources relating to the history of Portuguese-Jewish families: the De Pinto and Lopes Suasso family», Studia Rosenthaliana 32,2 (1998), p. 190-194. Jonathan I. Israel appelle Suasso «the archetypal big Jewish investor in colonial stock», dans «The Amsterdam Stock Exchange and the English Revolution of 1688», Tijdschrift voor Geschiedenis 103 (1990), 412440, voir p. 415.

${ }^{41}$ Voir les chiffres publiés par BLoom, The Economic Activities of the Jews of Amsterdam, p. 175-176.

${ }^{42}$ Daniel M. Swetschinski, The Portuguese Jewish Merchants of Seventeenth Century Amsterdam: A Social Profile (thèse dactylographiée, Brandeis University, 1980), p. 282. Une version condensée de cette thèse a été publiée sous le titre Reluctant Cosmopolitans: The Portuguese Jews of Seventeenth Century Amsterdam (London 2000), p. 140.

${ }^{43}$ Silva Rosa, Geschiedenis der Portugeesche Joden, p. 40; Jacob SASPORTas, Tsitsat Nôvel Tsvi, ed. Y. Tishbi (Jerusalem 1954), p. 260; Scholem, Sabbataï Tsevi, p. 430; KaPlan, «The Attitude of the Leadership», p. 203; ID., From Christianity to Judaism, p. 183-184. Gomes da Sousa mourut le 17 septembre 1667, selon l'épitaphe reproduit chez René KistemaKer \& Tirtsah Levie, Exodo. Portugezen in Amsterdam, 1600-1680 (Amsterdam 1988), p. 98.

${ }^{44}$ Swetschinski, The Portuguese Jewish Merchants, p. 396; ID., Reluctant Cosmopolitans, p. 194.

${ }^{45}$ Bloom, Economic Activities, 126; Swetschinski, Reluctant Cosmopolitans, p. 117. Sur cette maison et son commerce avec le Portugal, voir Swetschinski, The Portuguese Jewish Merchants, p. 201, 396; Jonathan I. IsRAEL, Empires and Entrepots: The Dutch, the Spanish Monarchy and the Jews, 1585-1713 (London 1990), p. 437. 
Il est plus difficile d'identifier les quatre autres personnes: Abraham Pimentel, Joseph Franco, Samuel Pinto et Jacob Netto, puisque ces noms sont assez fréquents parmi les juifs portugais. A l'époque où se situe l'affaire sabbataïste, on peut les retrouver, par exemple, parmi les étudiants du séminaire rabbinique d'Amsterdam ${ }^{46}$. Toutefois il est plus probable qu'Abraham Pimentel appartenait à la famille marchande des Abeniacar, que Samuel Pinto fut le négociant Francisco Vaz Pinto ${ }^{47}$ et que Jacob Netto est le courtier de change qui allait se convertir au christianisme quelque temps plus tard ${ }^{48}$. Jacob Netto et Joseph Nunes Franco sont attestés pendant les années 1640 au Brésil néerlandais ${ }^{49}$, et ce dernier fut le marchand le plus considérable parmi les mille cinq cent juifs qui y vécurent alors ${ }^{50}$.

L'examen des rapports du groupe avec le pouvoir communautaire nous réserve une surprise. Cinq parmi les quatorze personnes figurant sur la liste étaient très actives dans l'administration de la communauté ${ }^{51}$. João Nunes Henriques

${ }^{46}$ Abraham Cohen Pimentel, d'ailleurs sabbataïste fervent, enseignait à l'académie talmudique Keter Tora et publia en 1668 deux ouvrages religieux. Joseph Franco Serrano dit «de Suriname», élève et fils adoptif du rabbin Moïse Raphä̈l d'Aguilar, devint plus tard enseignant au séminaire Ets Haim (Mendes, «Memorias do estabelecimento», p. 63, 70; Silva Rosa, Geschiedenis der Portugeesche Joden, p. 67, 104). Tout comme lui, un Jacob Netto et un Samuel Pinto apparaissent en 1665 parmi les fondateurs de la confrérie caritative Tamimé Darekh. Le dernier fut en 1683 directeur de l'école religieuse Tora Or; voir Mendes, «Memorias do estabelecimento», p. 63; Wilhelmina Christina Pieterse, Daniel Levi de Barrios als geschiedschrijver van de PortugeesIsraelitiesche Gemeente te Amsterdam (Amsterdam 1968), p. 103, 110, 123, 187.

${ }^{47}$ Selon Vaz Dias dans le fichier cité. Un Samuel Pinto est attesté dans des transactions avec la Couronne d'Espagne; IsRaEl, Empires and Entrepots, p. 409.

${ }^{48}$ Swetschinski, The Portuguese Jewish Merchants, p. 475, 690-691; ID., Reluctant Cosmopolitans, p. 237.

${ }^{49}$ Hermann WätJEn, Das niederländische Kolonialreich in Brasilien (Berlin 1921), p. 128-129.

${ }^{50} \mathrm{Il}$ est rapporté qu'en 1654, peu de motivations ont autant animé les soldats portugais lors de la reconquête de Recife que l'espoir de piller sa maison; voir Arnold Wiznitzer, Jews in Colonial Brazil (New York 1960), p. 126, 174

${ }^{51}$ Le ma amad était constitué de sept hommes, à savoir six administrateurs (parnassim) et un trésorier (gabbaï); il fut renouvelé par moitié chaque année pour le nouvel an juif (en septembre) et le samedi précédant la Pâque. Ce conseil de sept, qui eut autorité aussi sur les rabbins, était élu non par suffrage universel, mais par une cooptation du conseil sortant. On avait toutefois limité le népotisme en ne permettant qu'une personne par famille à la fois et par la disposition selon laquelle un parnas ne pouvait être réélu qu'au bout de quatre ans. N'empêche qu'un groupe d'individus assez restreint avait réussi à occuper tour à tour la direction; voir SwETSCHINSKI, The Portuguese Jewish Merchants, p. 369, 376, 392; ID., Reluctant Cosmopolitans, p. 187-196; Jonathan I. IsRAEL, European Jewry in the Age of Mercantilism, 1550-1750 (Oxford 1985, 2éd. 1989), p. 197-198. 
-ou plutôt Abraham Nunes Henriques, comme il se fit appeler à la synagogue ${ }^{52}$ occupa, entre 1651 et 1698 , sept fois une place dans le conseil administratif de la communauté et cinq fois dans celui de l'école; son fils Jacob allait remplir ces mêmes dignités quatre fois entre 1702 et 1715 . Henrique -alias Isaac- Mendes da Silva ${ }^{53}$ fit partie du ma 'amad à neuf reprises entre 1661 et 1708 , tandis qu'il fut cinq fois administrateur de l'école. Lui aussi fut suivi dans ces fonctions par ses fils. Il se passe rarement une année où le grand clan des Abravanel da Sousa n'ait pas eu de représentant au ma 'amad ou au conseil de l'école, voire aux deux à la fois ${ }^{54}$. António Lopes Suasso alias Isaac Israël Suasso siégea, entre 1656 et 1673, quatre fois dans le conseil de la communauté et il fut une fois trésorier de l'école. L'année 1665 même, il avait été élu comme trésorier de la société de bienfaisance Bikur Holim ${ }^{55}$. Pour ce qui est des Coutinho mentionnés dans notre liste, Isaac Henriques Coutinho fit partie, en 1670, du conseil nommé pour la construction de la grande synagogue ${ }^{56}$.

Ces quatorze mécontents ne furent donc ni des sceptiques, ni des rebelles notoires, ni des laissés-pour-compte de la communauté judéo-portugaise d'Amsterdam. Tout au contraire, cette attaque violente contre l'autorité du ma 'amad est due à quelques individus parmi les mieux placés dans l'oligarchie communautaire et parmi les plus en vue dans les cercles religieux. Si ces hommes rappellent le texte du herem de Spinoza pour le retourner contre les autorités rabbiniques, leur bonne mémoire s'explique facilement: les malédictions fulminées contre Spinoza, ils les avaient jadis promulguées eux-mêmes. En effet Nunes Henriques et Lopes Suasso avaient personnellement fait partie du conseil qui décida l'exclusion du philosophe.

Le régime «aristocratique» en vigueur dans l'administration interne de la communauté judéo-portugaise est la réplique exacte de la Vroedschap, sénat coopté de trente-six membres qui gouvernait la ville d'Amsterdam; voir Henry Méchoulan, Amsterdam au temps de Spinoza: argent et liberté (Paris 1990), p. 58-59. Nous avons identifié les membres du conseil à partir des documents communautaires déposés aux Archives municipales d'Amsterdam.

${ }^{52}$ Délations d'André (Abraham) de Belmonte de 1661/62; AHN, Inq., lib. 1131, fol. 330vº.

${ }^{53} \mathrm{AHN}$, Inq., lib. 1131, fol. 327r ${ }^{\circ}$.

${ }^{54}$ Mentionnons Salomon et Elisée qui, entre 1641 et 1665, siégèrent chacun sept fois dans ces conseils. Samuel, fils d'Elisée, fut six fois entre 1678 et 1687 parnas de la communauté ou de l'école. Un autre Samuel, fils de Raphaël, remplit ces fonctions cinq fois entre 1665 et 1696, la dernière desquelles, il déclina la dignité. Siméon fut parnas six fois entre 1688 et 1711, année où il refusa également. Salomon, fils d'Isaac, occupa une de ces deux dignités huit fois entre 1690 et 1718; il mourut en fonction.

${ }^{55}$ Kaplan, From Christianity to Judaism, p. 166 note.

${ }^{56}$ Silva Rosa, Geschiedenis der Portugeesche Joden, p. 89. 
Mais il y a plus: en mai 1666, au moment où ils diffusèrent ce violent pamphlet contre les prérogatives juridiques du ma'amad, deux des «incroyants», à savoir Henrique Mendes da Silva et Antonio Lopes Suasso, firent eux-mêmes partie de l'autorité incriminée. Suasso avait réussi à se faire élire le 17 avril 1666, quelques jours seulement avant l'affaire du pamphlet, et Abraham Nunes Henriques avait fait partie des membres cooptants du conseil sortant. En bref, ces oligarques attaquent l'institution qu'ils représentent, en lui reprochant un acte d'intolérance qu'ils avaient eux-mêmes commis en son nom. Le jour où ils diffusent leur pamphlet, ils seront excommuniés par le ma 'amad, donc par euxmêmes. Quel sens y a-t-il à tout cela?

Assurément, aucun. Mais il semble que le petit pamphlet n'était pas du tout destiné à prendre parti dans les litiges communautaires. Il présente, en effet, un nombre d'éléments qui s'adaptent mal à la caricature du discours des autorités juives. Tout d'abord, le texte est rédigé en espagnol, langue internationale de l'époque, et non en portugais comme un document communautaire aurait dû l'être. Il va de soi qu'on chercherait en vain le mot «Jéhova» dans un décret lu à la synagogue -rien de plus contraire à la liturgie du judaïsme rabbinique que de prononcer le nom divin; en revanche, aucun lecteur d'une bible protestante ne douterait du fait que c'est bien ainsi que les Juifs appellent leur Dieu. En outre, les excommuniés ne figurent pas sous les noms hébreux qu'ils utilisaient parmi leurs coreligionnaires; le pasquin donne les noms espagnols ou même français dont ils se servaient dans les affaires. Deux parmi eux, les Sousa et les Carvalho, n'apparaissent que sous leurs sigles d'entreprise. Et finalement nous savons que ce texte ne fut pas distribué le vendredi soir à la synagogue, mais le lundi matin à la Bourse, et qu'il fut envoyé par la poste à des destinataires non-juifs ou en tout cas à des personnes bien éloignées de la communauté officielle, telle la société Montezinos Frères à Madrid. Notons que cette banque fut précisément l'entreprise correspondante par laquelle Henrique Mendes da Silva conduisit ses affaires espagnoles ${ }^{57}$. On doit conclure que la Liste était destinée aux partenaires commerciaux chrétiens, qui étaient considérablement inquiétés à l'époque par la perte de rationalité économique des marchands juifs pris dans la fièvre messianique ${ }^{58}$; elle devait les rassurer sur le fait que les quatorze entrepreneurs énumérés allaient rester solvables, fiables et dignes de crédit et qu'ils ne partageraient pas les réactions insensées d'autres entreprises juives, notamment des Curiel ou des Pereira, dont les capitaux, voire les chefs d'entreprise, avaient déjà pris le chemin de la Terre Sainte. Le pamphlet, on le devine, appar-

\footnotetext{
${ }^{57}$ Swetschinski, «The Spanish Consul», p. 171.

${ }^{58}$ Voir VAN WIJK, «Press Reports», p. 24-25.
} 
tient aux fameuses rumeurs de la Bourse d'Amsterdam ${ }^{59}$, négoce d'actualités qui ouvrait la semaine au marché des valeurs, car, selon le père Stanislas von Dunin-Borkowski, «les nouvelles du monde étaient concoctées le dimanche par les juifs d'Amsterdam pour être lâchées le lundi sur la Bourse» ${ }^{60}$. Les auteurs du pamphlet ont su canaliser et amplifier la diffusion de leur message en le vendant au lieu de le diffuser gratuitement. Détail bien adapté à la mentalité des gens d'affaires: ce qui a un prix a aussi une valeur.

La «liste des incroyants» nous permet de connaître les noms des principaux anti-sabbataïstes à Amsterdam. Compte tenu du document néerlandais dont nous parle Vaz Dias, il est possible que nous ayons affaire ici aux personnes qui avaient relâché leurs liens avec la synagogue; Suasso et Mendes da Silva, s'opposant à une majorité au sein du ma amad, ont pu choisir ce moyen pour se démarquer de ses décisions ${ }^{61}$. Mais si Suasso appartenait aux boycotteurs de la communauté, on comprend mal comment il a pu se faire coopter pour faire partie du ma 'amad encore en pleine fièvre messianique. En outre, un des Sousa qui figurent dans le pamphlet parmi les anti-sabbatéens, s'affiche comme «croyant» dans la lettre de l'académie talmudique déjà mentionnée ${ }^{62}$. Ces prétendus sceptiques, honnis par la synagogue dont ils calomnient l'autorité dans les termes les plus acerbes, menèrent-ils un double jeu?

Leur double jeu habituel, pourrait-on ajouter. Ce simulacre d'auto-excommunication relève bien de la comédie sociale du XVII siècle, et par l'ironie des choses, il nous ramène aussi vers le drame, souvent tragique, de la double religion, que Scholem crut devoir rendre responsable de l'esprit messianique. La plupart des quatorze «incroyants» avaient en effet des itinéraires personnels doubles ty-

\footnotetext{
${ }^{59}$ Joseph Penso de la Vega, un juif portugais, les peint sur le vif dans son livre Confusión de confusiones (Amsterdam 1688); voir Méchoulan, Amsterdam, p. 98. Les gens de la Bourse d'Amsterdam étaient célèbres pour l'attention qu'il dédiaient à la politique mondiale; IsRAEL, «The Amsterdam Stock Exchange and the English Revolution of 1688», p. 413.

${ }^{60}$ Stanislaus von Dunin-Borkowski, Der junge De Spinoza. Leben und Werdegang im Lichte der Weltphilosophie (Münster 1910), p. 151.

${ }^{61}$ Dans ce cas, les autres cinq dirigeants étaient possiblement des «croyants». Il s'agit de Salomon Salom alias António d'Azevedo (1613-1678), cinq fois parnas de la communauté et quatre fois de l'école entre 1647 et 1671, de David Henriques Faro alias Manuel Henriques Rodrigues, qui occupa l'une ou l'autre fonction sept fois entre 1655 et 1684, de Jacob Nunes Mendes, respectivement deux fois à la tête des deux institutions entre 1651 et 1667, puis de Moïse Gomes Porto alias Luís do Porto, qui n'a plus jamais été réélu. Le trésorier fut alors Jacob Belmonte, qui apparaît entre 1653 et 1670 cinq fois au ma 'amad de la communauté et trois fois au conseil administratif de l'école.
}

${ }^{62}$ Freimann, Sammelband Kleiner Schriften, p. 112-113. 
piques de la Nation portugaise. Ils étaient nés et baptisés chrétiens; et ils avaient tous vécu longtemps en Espagne avant d'embrasser le judaïsme à Amsterdam. S'ils poursuivaient leurs affaires espagnoles à partir de leur nouvelle patrie, ils se dérobaient aux yeux vigilants de l'Inquisition -toujours à la chasse des capitaux «juifs»- en dédoublant leur personnalité commerciale. Ainsi, João Nunes Henriques, installé à Madrid jusqu'à 1648 environ ${ }^{63}$, fut désormais Abraham Nunes Henriques à la synagoge et tantôt Diego de Araujo, tantôt Simón de la Estrella en Espagne ${ }^{64}$. Henrique Mendes da Silva, ancien Madrilène également, était le frère de Rodrigo Mendes da Silva, ci-devant chroniqueur de Philippe Iv, et le beau-frère de Diogo Henriques de Castro, pagador general de la Couronne espagnole installé à Anvers ${ }^{65}$; il utilisa selon les besoins les fausses identités de Jerónimo Rodríguez Pérez, Francisco Pérez ou Antonio Enríquez de Valle. António Lopes Suasso, né à Bordeaux, vécut à Cadix pendant les années $1640{ }^{66}$, d'où il vint se réfugier à Rouen vers 1648; il passa à Amsterdam en 1653. Ce serait simplifier les choses que de dire qu'il conduisit ses affaires espagnoles tantôt sous le nom de Giulio Bentivoglio, tantôt sous celui de Willem Vandenbergh ${ }^{67}$; en vérité Lopes Suasso partageait sa fausse identité italienne avec Jerónimo Nunes da Costa alias Moïse Curiel et la seconde, néerlandaise, avec Guillaume Mendes Sotto, un natif de Rouen connu également sous les noms de Cornelis Dirckszoon Cox, Jacob del Sotto ou Jacob del Monte ${ }^{68}$. Sebastião Coutinho signait ses cargaisons du nom d'Andries Mertens ${ }^{69}$, tandis que les frères Andrês \& Cristovão Nunes furent connus en Espagne sous le nom de Robert \& Henrik Mooyenbergh ${ }^{70}$. Excepté Diogo Nunes (alias Salomon Belmonte), le dernier

${ }^{63}$ AHN, Inq., lib. 1131, fol. 330v ${ }^{\circ}, 371 r^{\circ}$. En 1635, l'Inquisition de Cuenca accusa un homonyme réfugié à Anvers (AHN, Inq., leg. 449, no. 6294).

${ }^{64}$ Il exploitait notamment la route commerciale de la Baltique au Portugal; SwETSCHINSKI, The Portuguese Jewish Merchants, p. 175; ID., Reluctant Cosmopolitans, p. 123.

${ }^{65}$ AHN, Inq., lib. 1131, fol. $327 \mathrm{r}^{\circ}, 366 \mathrm{r}^{\circ}, 369 \mathrm{v}^{\circ}, 371 \mathrm{r}^{\circ}$.

${ }^{66}$ Ce détail, attesté dans les actes de l'Inquisition espagnole (AHN, Inq., lib. 1131, fol. 332r ${ }^{\circ}$; et lib. 1139 , fol. $317 \mathrm{r}^{\circ}$ ) doit être ajouté à l'étude biographique de Swetschinski et de Schönduve.

${ }^{67}$ Swetschinski et SchÖNDUve, De familië Lopes Suasso, p. 29-32.

${ }^{68}$ AHN, Inq., lib. 1131, fol. 332r ${ }^{\circ}$; voir sur cette famille Lydia SiJEs-Hagoort, «The Del Sottos, a Portuguese Jewish Family in Amsterdam in the Seventeenth Century», Studia Rosenthaliana 31 (1997), 31-57.

${ }^{69}$ SwETSCHINSKI, «The Spanish Consul», p. 169.

${ }^{70}$ Voir les délations d'Immanuel Lavello de 1655, dans SwETSCHINSKI, «The Spanish Consul», p. 168, et dans celles d'André (Abraham) de Belmonte de 1661/62; AHN, Inq., lib. 1131, fol. $333 \mathrm{r}^{\circ}, 336 \mathrm{r}^{\circ}, 338 \mathrm{v}^{\circ}, 340 \mathrm{r}^{\circ}-\mathrm{v}^{\circ}$. 
de notre liste, qui fut un Batave de naissance ${ }^{71}$, et les frères Carvalho, nés à Guarda et venus de Madrid en 1662 seulement ${ }^{72}$, la plupart des hommes de la «Liste» étaient arrivés à Amsterdam avec la grande immigration des années 1646-55: les uns d'Espagne, surtout de Madrid ${ }^{73}$, les autres -Joseph Franco et Jacob Netto- du Brésil néerlandais où, jusqu'à la reconquête portugaise de 1654, Moïse da Silva (alias Heitor Fernandes), les frères Sousa et les Senior Coronel avaient également établi leur commerce ${ }^{74}$.

Nos «incroyants» se sont ainsi recrutés dans les deux orientations économiques complémentaires des juifs néerlandais: les affaires avec la péninsule Ibérique d'une part et avec les Indes Occidentales d'autre part ${ }^{75}$. Ces deux versants de leur activité, la clandestinité toujours menacée et le triomphe colonial, ont été mis en rapport avec des convictions eschatologiques ${ }^{76}$. Mais la querelle sabbataïste d'Amsterdam nous montre que ni le labyrinthe des fausses identités ni les nouveaux horizons d'outremer n'ont prédisposé les juifs portugais à l'aventure messianique. Les «croyants» et les «incroyants» ne se distinguent guère dans les circonstances de leur vie personnelle, religieuse et sociale. Abraham (alias Tomás) Rodrigues Pereira et Abraham (alias João) Henriques Nunes, venus tous les deux de Madrid plus ou moins en même temps, dans les mêmes circonstances et à la même époque de leur vie, tout en partageant les mêmes occupations professionnelles, une égale fortune dans les affaires et des fonctions religieuses comparables, prirent des attitudes opposées dans la crise sabbataïste. L'《incroyant» António Lopes Suasso et le «croyant» Jerónimo Nunes da Costa allèrent jusqu'à devenir des agents pour les royaumes dont eux et leurs familles avaient été chassés ${ }^{77}$. Parmi les deux grands

${ }^{71}$ Il naquit à Amsterdam en 1621. Voir MendES, «Memoria do estabelecimento», p. 32; Ella Koen et al., «Notarial Records», Studia Rosenthaliana 6 (1972), p. 236 note.

${ }^{72}$ Cela suite à un procès inquisitorial, voir AHN, Inq., lib. 1131, fol. $331 \mathrm{v}^{\circ}$.

${ }^{73}$ Voir à propos de cette migration IsRAEL, Empires and Entrepots, p. 386-415, 436; cet auteur fait d'ailleurs un seul personnage des deux frères Andrês \& Cristovão Nunes.

${ }^{74}$ Swetschinski, The Portuguese Jewish Merchants, p. 176; ID., Reluctant Cosmopolitans, p. 115.

${ }^{75}$ Sur le conflit entre ces deux formes d'investissement et d'activité commerciale voir Jonathan I. IsRael, «The Trade of the Dutch Sephardim», dans Studies on the History of Dutch Jewry 4 (Jérusalem 1984), ed. J. Michman, p. 42.

${ }^{76}$ Jonathan I. IsRaEL, «Menasseh ben Israel and the Dutch Sephardic Colonization Movement of the Mid-Seventeenth Century (1645-1657)», dans Menasseh ben Israel and his World, éd. Y. Kaplan, H. Méchoulan et R. Popkin (Leiden 1989), p. 140-141; «Dutch Sephardi Jewry, Millenarian Politics, and the Struggle for Brazil (1640-1654)», dans Sceptics, Millenarians, and Jews, éd. D. S. Katz et J. I. Israel (Leiden 1990), p. 76-97.

77 À propos de Nunes da Costa et de sa famille, voir Daniel M. SwETsChinski, «An Amsterdam Jewish Merchant-Diplomat: Jeronimo Nunes da Costa (1620-1697), Agent of the King of 
promoteurs coloniaux de l'époque, João de Ilhão ${ }^{78}$ fut saisi par la fièvre et Joseph Franco garda la tête froide.

On ignore si la ligne de partage entre les uns et les autres était fonction de personnalités, d'attitudes familiales peut-être, de souci matériel; en tout cas, les indices sont trop partagés pour qu'on puisse attribuer les motivations du mouvement messianique à une aliénation religieuse ou à un malaise social qui serait particulier aux juifs portugais. Notre compréhension du sabbataïsme risque d'être faussée par un regard rétrospectif qui, trop conscient de la déception et de l'échec de ce mouvement, cherche ses origines dans une crise, voire dans une misère de la société juive -comme si l'attente messianique ne se fondait pas sur un contenu positif du judaïsme. Si le sabbataïsme se distingue radicalement des mouvements messianiques qui le précèdent, c'est qu'il réussit à saisir la diaspora juive dans sa totalité. Il se répandit grâce à la nouvelle fusion des aires culturelles du judaïsme qui fut acquise au milieu du XVII ${ }^{\mathrm{e}}$ siècle et dont témoignent tant l'intensification des échanges dans la vie matérielle que la nouvelle cohésion sur le plan doctrinal, créée par la diffusion et popularisation de la piété d'inspiration kabbaliste ${ }^{79}$. A la mise en place de ce nouveau réseau de liens commerciaux et intellectuels, les marchands et les imprimeurs judéo-portugais ont contribué d'une façon décisive, et cela précisément vers le milieu du siècle. On doit à François Secret la découverte d'un document éloquent faisant cas de leur présence à Smyrne même, où se manifesta Shabbataï Sevi ${ }^{80}$.

L'apport des juifs portugais à la diffusion du mouvement se situe probablement dans ce rôle de médiateurs et non dans le fait d'avoir contaminé la diaspora

Portugal», dans Neveh Ya'akov. Jubilee Volume Presented to Dr. Jaap Meijer, éd. L. Dasberg et J. N. Cohen (Assen 1982), p. 3-30; Jonathan I. IsRaEL, «An Amsterdam Jewish Merchant of the Golden Age: Jeronimo Nunes da Costa (1620-1697), Agent of Portugal in the Dutch Republic», Studia Rosenthaliana 18,1 (1984), 21-40; Edgar R. SAmuEL, «The Curiel Family in 16th-Century Portugal», Jewish Historical Studies 31 (1990), 111-136; Richard Ayoun, «Jerónimo Nunes da Costa, un diplomate et financier entre Amsterdam et le Portugal au XVII siècle», dans 1492. L'expulsion des juifs d'Espagne, éd. R. Goetschel (Paris 1996), p. 111-119; Michael STUDEMUNDHalévy, Biographisches Lexikon der Hamburger Sefarden (Hamburg 2000), p. 379-384.

${ }^{78}$ João d'Ilhão avait en 1651 la concession commerciale pour Curaçao; voir Isaac S. et Suzanne A. Emmanuel, History of the Jews in the Netherlands' Antilles (Cincinnati 1970), t. I, p. 40-42. Dix ans plus tard, il proposa ses services à Louis xIV; voir Zvi LOKER, «Juan de Yllán, Merchant Adventurer and Colonial Promotor. New Evidence», Studia Rosenthaliana 17 (1983), p. 22-31.

${ }^{79}$ Cette nouvelle «cohésion» est soulignée par IsRaEL, European Jewry in the Age of Mercantilism, p. 3, 33, 71 .

${ }^{80}$ François SeCRET, «Judaica oubliés, II: Juifs de France à Smyrne, et l'aventure de Juan de la Paz», Hommage à Georges Vajda (Louvain 1980), p. 380-384. 
par un malaise identitaire. Le jeune Scholem et ses successeurs, piégés par des problématiques plus récentes, ont jumelé a priori la duplicité religieuse avec l'urgence de parvenir à l'intégrité perdue -par des miracles ou des fantasmagories si nécessaire ${ }^{81}$. Quant aux auteurs de la dérision calculée des postures messianiques, ils se sont tranquillement resservi d'une technique adroite d'attitudes religieuses contrefaites, pour empêcher que les troubles du sentiment religieux ne perturbent leur existence au sein de cette rationalité de l'échange financier qui, comme Henry Méchoulan nous l'a montré, fondait les relations entre les croyants des différentes communautés dans l'Amsterdam du temps de Spinoza. Celui-ci, nous l'avons vu, est présent en filigrane dans la querelle qui divisa ses anciens coreligionnaires. Retiré à Rijnsburg, il écrivit pendant ces mois son Traité théologico-politique. Si les miracles existaient, note-t-il, ils devraient nous faire douter de Dieu, car ils ne pourraient que démentir Son action dans le cours de ce monde. Dans ce monde, les cours des actions seraient alors en péril: inutile de l'apprendre aux professionnels de l'argent.

\section{RESUMEN}

Según una tesis ideada por Gershom Scholem, el movimiento mesiánico de Sabetay Sebí debía su éxito a la atracción que su mensaje paradójico ejerció entre los conversos vueltos al judaísmo en el siglo XVII. Con intención de criticar esta explicación, este artículo presenta y analiza un panfleto satírico recién descubierto que había sido distribuido por el partido anti-sabataísta de la comunidad judeoportuguesa de Ámsterdam el 3 de mayo de 1666, cuando llegó la noticia de la prisión de Sabetay Sebí. Este texto demuestra que varias de las más prósperas e influyentes cabezas de la comunidad no creían en el falso mesías. Enfrentándose al partido sabataísta, estos «incrédulos» emplearon estrategias de engaño onomástico y literario semejantes a las que practicaban en sus relaciones con la España católica. La herencia criptojudaica peninsular no les inspiraba, pues, ningún entusiasmo místico; al contrario, se ven preocupados, más que nada, por conservar la reputación de racionalidad y confianza de la cual gozaban en el mundo europeo de las finanzas. Su polémica incluye una alusión irónica a las sanciones que la comunidad de Ámsterdam había impuesto a sus herejes Uriel da Costa y Bento Espinoza.

Palabras ClaVe: Judíos portugueses, Ámsterdam, marranismo, Sabetay Sebí.

${ }^{81}$ Michael Löwy, Rédemption et utopie: le judaïsme libertaire en Europe centrale. Une étude d'afinnité élective (Paris 1988); ID., «Le messianisme hétérodoxe dans l'œuvre de Gershom Scholem», dans Messianismes: variations sur une figure juive, éd. Jean-Christophe Attias et al. (Genève 2000), p. 131-145. 


\section{SUMMARY}

According to an opinion voiced by G. Scholem, the messianic movement of Shabbatai Zevi owed much of its success to the attraction that his paradoxical message exerted among Conversos returning to Judaism in the $17^{\text {th }} \mathrm{C}$. With the intention of criticizing this explanation, the author presents and analyzes in this article a recently discovered satyrical pamphlet that was distributed by the anti-Shabbatean party in Amsterdam following the arrival of news concerning Shabbetai's prison on May 3, 1666. This text shows that some of the more influential and prosperous Jewish communal leaders did not believe in the false Messiah. Confronting the Shabbatean party, these "unbelievers" used tactics of literary deceit similar to those used in their relation to Catholic Spain. Their Iberian Marrano origins did not arouse in them any mystical enthusiam. To the contrary, they were concerned with keeping the reputation of rationality and trust they had enjoyed in the European world of finances. This controversy includes an ironical reference to the sanctions that the Amsterdam Jewish community imposed on their heretics Uriel da Costa and Baruch Espinoza.

KEYwords: Portuguese Jews, Amsterdam, Marranism, Shabbatai Zevi. 